\title{
Hemophagocytic Lymphohistiocytosis after Severe Fever with Thrombocytopenia Syndrome Virus Infection
}

Q. C. WANG AND YUYU LU ${ }^{1 *}$

School of Clinical Medicine, Nanjing Medical University, Nanjing, Jiangsu 210029, China, ${ }^{1}$ Department of ICU, The Affiliated Jiangning Hospital of Nanjing Medical University, Nanjing, Jiangsu 210029, China

Wang and Lu et al.: Hemophagocytic lymphohistiocytosis with thrombocytopenia syndrome

This investigation reports of a case of hemophagocytic lymphohistiocytosis caused by severe fever with thrombocytopenia syndrome bunya virus, a novel virus first described in 2011. This patient presented with high fever and laboratory study revealed the presence of thrombocytopenia. Further laboratory examinations confirmed the coexistence of severe fever with thrombocytopenia syndrome and hemophagocytic lymphohistiocytosis. Review of relevant publications led to a proposal that severe fever with thrombocytopenia syndrome as a trigger of hemophagocytic lymphohistiocytosis. More 
attention should be paid to severe fever with thrombocytopenia syndrome associated hemophagocytic lymphohistiocytosis and further studies be conducted.

Key words: Hemophagocytic lymphohistiocytosis, thrombocytopenia syndrome, bunya virus, severe fever with thrombocytopenia syndrome virus (SFTSV)

Hemophagocytic lymphohistiocytosis (HLH) is a hyper inflammatory syndrome with high mortality that occur in many underlying conditions and in all age groups ${ }^{[1,2]}$. Hyper activation and proliferation of $\mathrm{CD} 8{ }^{+} \mathrm{T}$ cells and macrophages, infiltration of these cells into various organs as well as hypercytokinemia, result in organ damage that may lead to death. $\mathrm{T}$ cells play a crucial role in the HLH pathogenesis, but their pattern of differentiation is not obviously in patients with active $\mathrm{HLH}^{[3]}$. The major symptoms are prolonged high fever, hepatospleenomegaly, cytopenia, neurologic dysfunction, liver dysfunction, cytopenia, hypertriglyceridemia, hyperferritinemia, hemophagocytosis and diminished NK cell activity ${ }^{[4]}$. According to different etiologies, HLH can be divided into familial HLH and acquired HLH. Acquired HLH typically happens in older children and adults. There are a variety of causes for acquired HLH, among which infection is the most common one. Herpes virus, especially Epstein-Barr virus $(\mathrm{EBV})^{[5,6]}$, is the most common organism that has been implicated in acquired HLH. There is no single criteria that is specific for diagnosis of HLH including hemophagocytosis and diagnosis of HLH is often delayed because of rare occurrence, variable presentation and nonspecific findings ${ }^{[7]}$. Lymphocytic tumors account for a large part of malignant tumour-related HLH, among which $\mathrm{T}$ or NK cells lymphoma is the most common ${ }^{[8]}$. Long term morphological observation showed that the morphologic characteristics of the benign proliferation of lymphoma cell nucleus in some patients with bone marrow infiltration were very similar, which could not be easily distinguished by microscopy alone. Especially in patients with multiple lymphadenopathies, long term fever and other symptoms. Careful observation of the morphology of bone marrow cells and combining with other laboratory findings, one can preliminarily judge whether HLH is caused by severe fever with thrombocytopenia syndrome viral (SFTSV) infection or the HLH is related to lymphoma. Familial HLH is an inherited version of the disease that occurss during infancy and early childhood ${ }^{[9]}$. It is estimated that one

*Address for correspondence E-mail: cllyuwan@163.com in every 50000 children may develop familial HLH. HLH may also cause hemophagocytosis and affected patients have low numbers of red blood cells and platelets. The reduction of platelets may cause easy bruising and abnormal bleeding ${ }^{[10]}$. HLH should be distinguished and treated as soon as possible to access to the best possible treatment. This disease is usually identified by specialized blood tests in clinical terms. There are no clear guidance for HLH treatment, but scientists and doctors have developed a series of methods based on the underlying cause and severity of symptoms. If the disorder is suspected, even if all diagnostic criteria are not met with, treatment of HLH should be started. Paediatric haematologist should take the prime responsibility for the disease. Treatments rely on the occurrence of some factors such as a family history of HLH, coexisting infections and demonstrated immune system defects. Therapeutic options include immunosuppressive, immunomodulatory and cytotoxic drugs. Children with familial HLH ordinarily accept allogeneic stem cell transplantation to replace their defective immune system with a healthy one. So early discovery of HLH and assessment of potential causes are vitally important ${ }^{[11]}$. Stem cell transplantation is required to cure genetic HLH. Severe fever with thrombocytopenia syndrome (SFTS) is an emerging infectious disease transmitted by tick bites that was first described in rural areas in China. It was a syndrome with clinical and epidemiological similarity to human anaplasmosis. Most cases occurred in rural areas with rich vegetation and humid climate in middle and low altitude mountains and hills. May to September might be the high incidence time and patients are mostly 40$80 \mathrm{y}$ old farmers. The incidence rate and prevalence of SFTS increases year after year, but mortality of patients is gradually decreasing. The case fatality rate of SFTS in China has decreased from 12 to $30 \%$ at present, which is mainly benefited from the improvement of diagnostic methods and the improvement of treatment level of clinicians. SFTSV, a novel phlebovirus that belongs to the family Bunyaviridae, has been identified as the causative agent for SFTS ${ }^{[12]}$. However, it was also found that human to human transmission was caused by contact with body fluids of patients. The symptoms of SFTS are relatively nonspecific. 
Three primary stages of disease have been identified, the fever stage, the multiorgan dysfunction stage and the convalescent stage. Days 1 to 7 were generally regarded as the fever period. The second stage appears from days 7 to 13 in nonfatal cases. In the third stage, fatal cases develop in to multiorgan dysfunction, disseminated intravascular coagulation, overt haemorrhage and death. Major onset symptoms include fever and gastrointestinal symptoms, which are followed by progressive decline in platelet and white blood cell levels as seen in this patients. Unilateral inguinal lymphadenopathy with found in this patient. The key characteristics of SFTSV infection and death are the continuous replication of the virus and the severe reduction of platelets. However, the underlying pathogenesis of SFTSV is still unknown. Therefore, only the patients with this disease can receive universal supportive treatment specific effective treatment ${ }^{[13]}$. In order to understand the pathogenesis of SFTS and the role of host immune defence in the process of virus infection, as well as to develop effective prevention and treatment methods. It is necessary to study the immune mechanism of SFTSV infection, especially the regulation of specific humoral immune response, the relationship with prognosis of patients and clarify the immunological mechanism. Interferon $\beta$ (IFN- $\beta$ ) production is a defence mechanism of host innate immune system against viral infection. However, IFN- $\beta$ can hardly be detected in the blood of patients with SFTS. In the monocytes infected with SFTSV, the transcription factors related to IFN- $\beta$ were upregulated, but the downstream molecular levels of TNF receptor-related factors 3 and 6 and mitochondrial antiviral signal protein were not changed or downregulated, so the induction of IFN- $\beta$ was inhibited. Laboratory tests showed elevated APTT, serum alanine aminotransferase (ALT), AST, LDH, CK and creatine kinase $\mathrm{MB}$ fraction (CK-MB), which suggest multiple organ injury. The clinical outcome of SFTS is quite variable, some patients experienced self-limiting clinical outcome, but for the others fatal outcome is not avoidable. The case data was originated from the affiliated Jiangning Hospital of a Nanjing Medical University. General data were collected, including age, gender, history of tick bite, total fever time, maximum fever temperature, previous history, symptoms and signs. During the treatment of patient, we had a routine bone marrow puncture, then smear and had a WrightGiemsa staining for the bone marrow cells. In addition to routine laboratory tests such as routine blood picture, tenderness is frequently observed in SFTS, although not of viral infection in clinical practice and there is no

liver function, kidney function, myocardial enzyme spectrum, serum amylase, coagulation function, stool and urine analysis, $\mathrm{T}$ cell subsets detection, C-reactive protein, procalcitonin, ECG, CT and MRI, the SFTSV RNA and its specific IgM antibody in the plasma was tested. Combined with the epidemiological history, clinical manifestations, comorbidities, complications, physical examination and laboratory examination results of the patients, analysed the risk factors of death. The clinical diagnosis standard refers to the prevention and treatment guide of fever with thrombocytopenia issued by the general office of the Ministry of health in 2010. The present report is related to a female patient, who presented with nausea, vomiting and fever at the local hospital. Her temperature rose to $39-40^{\circ}$ and the laboratory findings revealed platelet count of $73 \times 10^{9} / 1$, which three days later decreased to $25 \times 10^{9} / 1$. She was transferred to another hospital, after which the patient appeared to be somnolent, her laboratory results are displayed in Table 1 and the cranial computed tomography (CT) and magnetic resonance imaging (MRI) revealed no specific findings. Acute leukaemia was suspected by a haematologist in the hospital. So, the patient was transferred to the department of emergency in this hospital with consideration of severity of this disease. The patient has a history of chronic bronchitis and diabetes. The patient lived in a forest farm, her family reported that the patient did not have any insect bites, but they reported that several people around had been bitten by ticks in recent months and most of them had similar symptoms including fever and

\section{TABLE 1: LABORATORY RESULTS OF THE FEMALE} PATIENT

\begin{tabular}{lc}
\hline Index & Patient \\
\hline Gender & Female \\
White cell & $2.0 \times 10^{9} / \mathrm{l}$ \\
Neutrophil & $1.6 \times 10^{9} / \mathrm{l}$ \\
Haemoglobin & $118 \mathrm{~g} / \mathrm{l}$ \\
Urinary protein & +++ \\
Total protein & $47.2 \mathrm{~g} / \mathrm{l}$ \\
Albumin & $25.2 \mathrm{~g} / \mathrm{l}$ \\
Globulin & $25.2 \mathrm{~g} / \mathrm{l}$ \\
Aspartate aminotransferase (AST) & $130 \mathrm{U} / \mathrm{l}$ \\
Lactate dehydrogenase (LDH) & $1672 \mathrm{U} / \mathrm{l}$ \\
Creatine kinase (CK) & $249 \mathrm{U} / \mathrm{l}$ \\
Urea & $7.8 \mathrm{mg} / \mathrm{dl}$ \\
Glucose & $12.6 \mathrm{mM}$ \\
C-reactive protein (CRP) & $<0.5 \mathrm{mg} / \mathrm{l}$ \\
Prothrombin time (PT) & $15.2 \mathrm{~s}$ \\
International normalized ratio (INR) & 1.3 \\
activated partial thromboplastin time (APTT) & $60.8 \mathrm{~s}$ \\
Fibrinogen & $1.05 \mathrm{~g} / \mathrm{l}$ \\
D-dimer & $9.13 \mathrm{mg} / \mathrm{l}$ \\
\hline
\end{tabular}


thrombocytopenia. On examination, the patient appeared pale and somnolent. The temperature was $37.6^{\circ}$, blood pressure was $140 / 67 \mathrm{~mm} \mathrm{Hg}$, pulse was 85 beats/min and the oxygen saturation $90 \%$ while she was breathing ambient air. Small ecchymoses were noted on her left arm and abdominal wall, further palpation revealed no lymphadenopathy and hepatospleenomegaly. Considering her possible exposure to tick bites, a blood sample was sent to Center for Disease Control to test severe fever with thrombocytopenia syndrome (SFTS) virus, which is transmitted by tick bites. Simultaneously, a bone marrow puncture was performed to exclude the possibility of an underlying haematological disease. Ribavirin and cefoperazone was administered along with injection of granulocyte colony stimulating factor (G-CSF). Despite the above therapy, the patient became worse and on the second hospital day the patient became unconscious. A lumbar puncture was performed to exclude an intracranial infection and results of cerebrospinal fluid revealed slightly elevated protein $(0.52 \mathrm{mM})$ and glucose $(6.00 \mathrm{mM})$, other results turned out to be normal. CT of chest was then performed, which revealed nonspecific findings that suggested pneumonia. On the third hospital day, bone marrow examination revealed histiocytic hyperplasia with prominent hemophagocytosis (fig. 1). Further laboratory studies were as follows, ferritin $>15000 \mathrm{ng} /$ $\mathrm{ml}$, triglyceride $3.76 \mathrm{mM}$, soluble CD25 (sCD25) 6188 ng/l (reference: normal <2000 ng/l). Besides, plasma was positive for SFTSV RNA and its specific IgM antibody.

Combining these results, a diagnosis of HLH associated with SFTSV infection was established. The patient became worse, 0xygen saturation was $83 \%$ on high flow oxygen mask (10 1/min). Because of

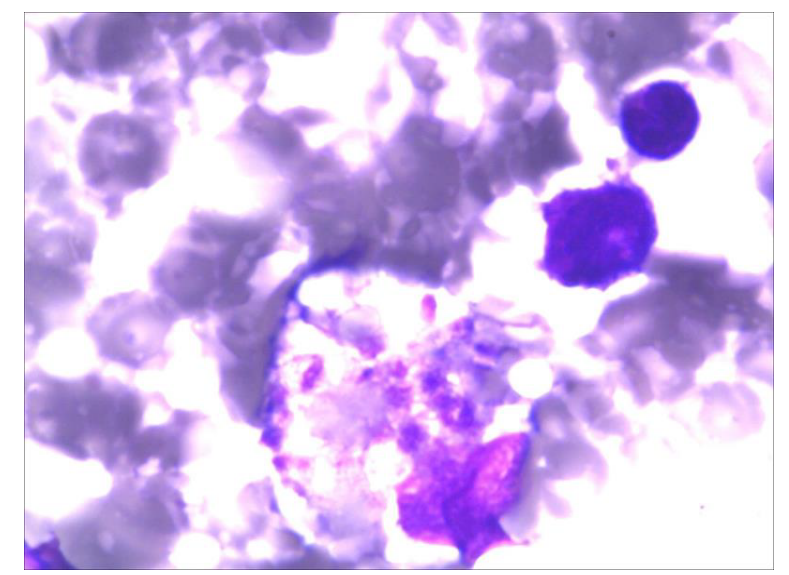

Fig. 1: Bone marrow examination

Bone marrow examination revealed prominent phagocytosis of mature red blood cells and platelets by phagocytes worsened hypoxia, the patient was intubated to provide mechanical ventilation. Methylprednisolone $200 \mathrm{mg}$ and $20 \mathrm{~g}$ intravenous immunoglobulin (on the first day, the dose of immunoglobulin was $10 \mathrm{~g}$ for financial reason) was initiated. The patient was transferred to the intensive care unit but finally died of progressive respiratory failure. HLH is characterized by highly stimulated but ineffective immune response. Defects in NK cells and T cells result in cytokine release and aggravation of inflammation response ${ }^{[14]}$. HLH can be generally classified into two types, familial HLH or acquired HLH on the basis of different underlying etiologies. Mutations in genes involved in the cytolytic secretory pathway that cause perforin and granzyme to induce apoptosis upon target cells may lead to familial HLH. Acquired HLH is most commonly triggered by infection, followed by malignancies, rheumatologic diseases and immune deficiency syndromes ${ }^{[15]}$. The predominant clinical findings of HLH included fevers, cytopenia, hepatitis and splenomegaly ${ }^{[16]}$. The histiocyte society proposed a standard definition of HLH in 1994 which was later modified for the HLH-2004 trial ${ }^{[17]}$. According to revised diagnostic guidelines during the HLH-2004 trial, this patient met 6 out of 8 diagnostic criteria for HLH, including fever, bicytopenia, hyperferritinemia, hyperlipidemia or/and hypofibrinogenemia and elevated level of sCD25. Diagnosis of SFTSV infection associated HLH was established after confirming the virus infection by special antibody and RNA testing. SFTS should be taken into consideration in patients with a syndrome characterized by acute fever (temperature $\geq 38^{\circ}$ ), thrombocytopenia (PLT $<100 \mathrm{~K} / \mathrm{l})$ and leukopenia $(\mathrm{WBC}<2.5 \mathrm{~K} / \mathrm{l})$, expressly in direct contact with a history of tick exposure/bite in an endemic area. Several cases of HLH associated with SFTS have been reported in recent years ${ }^{[18-21]}$. All the case reports were published in Chinese. Out of these cases, 5 cases have details available for review ${ }^{[18-20]}$. In these cases, the clinical course of 2 cases seem to be self-limiting, with only supportive care. One case accompanied by Anaplasma phagocytophilum infection eventually succumbed to multiple organ failure ${ }^{[18]}$. The pathological mechanisms underlying SFTSV-associated HLH is still not clear. NK cells were found to increase in acute phase and severe SFTS $^{[22]}$. NK cells secreted cytokines like IFN- $\gamma$, TNF- $\alpha$, IL-10. It was reported that the level of INF- $\gamma$ increased in severe SFTS and in some severe cases a cytokine storm could be observed ${ }^{[23]}$. So it is postulated that hypercytokinemia or cytokine storm might contribute to development of HLH in SFTS. 
Furthermore, according to a recent study, SFTSV can adhere to platelets, which are phagocytosed by splenic macrophages, this phenomenon may partially explain the phagocytosis in SFTSV-associated $\mathrm{HLH}^{[24]}$. Through the examination of bone marrow cytology smear, one can see the obviously increase of histiocytes in different degrees, and the phenomenon of phagocytic histiocytes phagocytizing mature erythrocytes, platelets and granulocytes. The impact of HLH on prognosis of SFTS remains to be determined, based on available literature, 4 of 19 cases died, with a mortality rate of $21.1 \%$ similar to that of SFTS patients in a recent study ${ }^{[25]}$. So far, there have been no relevant studies about treatment of HLH associated with SFTS bunyavirus. Treatment of HLH emphasizes on suppression on overwhelmed immune reaction and underlying hypercytonemia. Furthermore, therapy for secondary HLH should be directed at treating the underlying cause because HLH can recover by the treatment of the underlying disease or infection, in this regard, bunyavirus infection should be treated in patients suffering HLH associated with SFTS bunyavirus. But until now, there is no specific treatment for SFTS, supportive care should be given as soon as possible. Ribavirin, an antiviral agent, which has been approved for the treatment of other bunyavirus infections including Rift Valley fever virus and Crimean-Congo haemorrhagic fever virus, may have limited clinical efficacy in treating SFTSV infection ${ }^{[12]}$. Intravenous immunoglobulin is an appropriate adjunct therapy for most viral infections ${ }^{[7]}$. There is no evidence that corticosteroids are effective in treatment of SFTSVassociated HLH and immunosuppressive therapy with corticosteroid should be initiated with caution because corticosteroids may promote the replication of SFTSV leading to aggravation of the condition of the patient. Based on HLH-2004, etoposide-containing regimens are recommended for patients with HLH, with or without evidence of genetic or familial disease ${ }^{[17]}$. In one reported case, the patient was treated by etoposidebased regimen, the doses was rapidly tapered after the testing for bunyavirus turned negative and the patient recovered after the completion of the therapy. But it remains unclear that whether the survival of this patient should be attributed to the therapy or just the self-limiting nature of this disease. There is limited experience with this special clinical condition. The incidence of SFTSV-associated HLH remains to be determined. It is postulated that the incidence of SFTSV-associated HLH may be underestimated in China as patients with SFTS are usually admitted to the department of infectious disease where tests for HLH are not routinely adopted in the diagnosis and evaluation of this disease. It is not uncommon for SFTS to be associated with HLH, especially for patients with severe hemocytopenia and multiple organ dysfunctions. If sHLH is combined with SFTS and there are no obviously effects, antisHLH treatment should be considered and put a high premium on SFTS related HLH may be helpful to reduce the mortality of the disease. The underlying mechanisms and detailed clinical characteristics of SFTSV associated $\mathrm{HLH}$, as well as related therapy, are still unclear. As a new infectious disease with strong infectivity and high mortality, SFTS has gradually become a serious public health problem. At present, there is no specific treatment for SFTS and the most important measure to prevent SFTSV infection and transmission is to prevent tick bites. When rescuing or nursing critically ill patients, especially when the patients have hemolysis, hematemesis and other bleeding problems, medical personnel and accompanying personnel should strengthen personal protection to avoid direct contact with the patient's blood. The pathogenesis and treatment of SFTS needs further study. There were some SFTS patients also meet HLH clinical diagnostic criteria and the progression of SFTS disease is likely to be closely related to HLH caused by novel bunyavirus infection. Since the rapid progression of HLH disease, early diagnosis is particularly important. Although it is not validating whether there is an inevitable link between SFTS and HLH, and the pathogenesis is unknown. It can lead clinicians to make further differential diagnosis of related diseases from the cases. This paper reports a case analysis, and it is essential that further research is needed involving many such cases. Many issues between HLH and SFTSV needs to be explored and greater efforts to improve diagnosis and treatment as well as understanding of this emerging disease should be made.

\section{Author's contributions:}

Yuyu $\mathrm{Lu}$ designed experiments and analysed experimental results and analysed sequencing data. Qiangcheng Wang wrote the manuscript.

\section{Ethical approval:}

All studies were reviewed by the academic ethics committee of the affiliated Jiangning Hospital of a Nanjing Medical University review board. Informed consent was obtained from the patient's family for publication of this case report and any accompanying images. A copy of the written consent is available for review by the editor of this journal. 


\section{Conflict of interest:}

All authors report no conflicts of interest in this work.

\section{REFERENCES}

1. Weitzman S. Approach to hemophagocytic syndromes. Haematology Am Soc Hematol Educ Program, 2011;2011:17883.

2. Canna SW, Marsh RA. Pediatric hemophagocytic lymphohistiocytosis. Blood 2020;135:1332-43.

3. Ammann S, Lehmberg K, zur Stadt U, Janka G, Rensing $\square$ Ehl A, Klemann C, et al. Primary and secondary hemophagocytic lymphohistiocytosis have different patterns of T-cell activation, differentiation and repertoire. Eur J Immunol 2017;47:364-73.

4. Melissa RG. Hemophagocytic lymphohistiocytosis: review of etiologies and management. J Blood Med 2014;5:69-86.

5. Mascia G, Argiolas D, Carta E, Ibba S, Piredda GB. Hemophagocytic Lymphohistiocytosis in Renal Transplant Recipients: A 2-Case Report. Transplant Proc 2020.

6. Gomez R, Maakaron J, Baiocchi R. Macrophage Activation Syndrome Versus Hemophagocytic Lymphohistiocytosis: A Diagnostic Dilemma in a Patient With Still's Disease and Epstein-Barr Virus Viremia. J Hematol 2019;8:68-70.

7. Jordan MB, Allen CE, Weitzman S, Filipovich AH, McClain KL. How I treat hemophagocytic lymphohistiocytosis. Blood 2011;118:4041-52.

8. Konkol S, Rai M. Lymphohistiocytosis (Hemophagocytic Lymphohistiocytosis), in StatPearls 2020.

9. Yeşilbaş O, Köker A, Şevketoğlu E. Propionic acidemia: an extremely rare cause of hemophagocytic lymphohistiocytosis in an infant. Arch Argent Pediatr 2020;118:e174-7.

10. Filipovich AH. The expanding spectrum of hemophagocytic lymphohistiocytosis. Curr Opin Allergy Clin Immunol 2011;11:512-6.

11. Allen CE, McClain KL. Pathophysiology and epidemiology of hemophagocytic lymphohistiocytosis. Haematology Am Soc Hematol Educ Program 2015;2015:177-82.

12. Liu Q, He B, Huang SY, Wei F, Zhu XQ. Severe fever with thrombocytopenia syndrome, an emerging tick-borne zoonosis. Lancet Infect Dis 2014;14:763-72.

13. Li XK, Lu QB, Chen WW, Xu W, Liu R, Zhang SF, et al. Arginine Deficiency Involved in Thrombocytopenia and Immunosuppression in Severe Fever with Thrombocytopenia Syndrome. Sci Transl Med 2018;10.

14. Usmani GN, Woda BA, Newburger PE. Advances in understanding the pathogenesis of HLH. $\mathrm{Br} \mathrm{J}$ Haematol 2013;161:609-22.
15. Devitt K, Cerny J, Switzer B, Ramanathan M, Nath R, Yu $\mathrm{H}$, et al. Hemophagocytic lymphohistiocytosis secondary to T-cell/histiocyte-rich large B-cell lymphoma. Leuk Res Rep, 2014;3:42-5.

16. Filipovich AH. Hemophagocytic lymphohistiocytosis (HLH) and related disorders. Hematology Am Soc Hematol Educ Program 2009;2009:127-31.

17. Henter JI, Horne A, Aricó M, Egeler RM, Filipovich AH, Imashuku S, et al. HLH-2004: Diagnostic and therapeutic guidelines for hemophagocytic lymphohistiocytosis. Pediatr Blood Cancer 2007;48: 124-31.

18. Jianning W, Qingqi M, Min S, Liubo S, Yanqiu H, Hongyu B. Hemophagocytic lymphohistiocytosis after severe fever with thrombocytopenia syndrome bunyavirus infection: two cases and literature review. Chin J Gen Pract 2013;12:3.

19. Lingling WH, Weifeng C, Wei Z, Ji X, Hongxia Q, Jianyong L. Novel bunyavirus infection associated hemophagocytic lymphohistiocytosis: two cases. Chin J Hematol 2011;33:250.

20. Nian C, Jun L. Novel bunyavirus infection associated hemophagocytic lymphohistiocytosis. Chin J Clin Infect Dis 2011;4:269-270.

21. Yunjin ZL, Yu L, Zhangzhi L, Bin S, Jia H. Novel bunyavirus infection associated hemophagocytic lymphohistiocytosis: clinical analysis of 12 cases J Clin Intern Med 2013;30:487-8.

22. Sun L, Hu Y, Niyonsaba A, Tong Q, Lu L, Li H, et al. Detection and evaluation of immunofunction of patients with severe fever with thrombocytopenia syndrome. Clin Exp Med 2014;14:389-95.

23. Sun Y, Jin C, Zhan F, Wang X, Liang M, Zhang Q, et al. Host cytokine storm is associated with disease severity of severe fever with thrombocytopenia syndrome. J Infect Dis 2012;206:1085-94

24. Jin $\mathrm{C}$, Liang $\mathrm{M}$, Ning $\mathrm{J}, \mathrm{Gu} \mathrm{W}$, Jiang $\mathrm{H}, \mathrm{Wu} \mathrm{W}$, et al. Pathogenesis of emerging severe fever with thrombocytopenia syndrome virus in C57/BL6 mouse model. Proc Natl Acad Sci 2012;109:10053-8.

25. Gai ZT, Zhang Y, Liang MF, Jin C, Zhang S, Zhu CB, et al. Clinical progress and risk factors for death in severe fever with thrombocytopenia syndrome patients. J Infect Dis 2012;20:1095-102.

This is an open access article distributed under the terms of the Creative Commons Attribution-NonCommercial-ShareAlike 3.0 License, which allows others to remix, tweak, and build upon the work non-commercially, as long as the author is credited and the new creations are licensed under the identical terms

This article was originally published in a special
issue, "Biomedical Research in Healthcare Setting"
Indian J Pharm Sci 2020:82(3)Spl issue5;71-76

